\title{
Issues and contributions in interactive multimedia: photos, mobile multimedia, and interactive TV
}

\author{
Ethan V. Munson • Maria da Graça C. Pimentel
}

(C) Springer Science+Business Media, LLC 2011

\section{Editorial process}

We solicited papers from a variety of research groups active in the area of interactive multimedia. After a rigorous reviewing process, in which each paper was reviewed multiple times by multiple reviewers, we selected seven for inclusion in this special issue.

\section{Selected papers on interactive multimedia}

The seven papers selected discuss issues and present contributions related to managing and manipulating digital photographs, adapting multimedia content to mobile devices, and supporting interactive television.

Ryu, Chung, and Cho's paper is titled "A Hierarchical Photo Visualization System Emphasizing Temporal and Color-based Coherences" and presents a novel user interface for visualizing photo collections. Their system presents photos on a grid that is organized both by the time at which the photos were taken and by color metrics, rather than using the more obvious graph presentation [5]. Groups of similar photos are represented on the grid by a

\footnotetext{
E. V. Munson $(\bowtie)$

Department of EECS, University of Wisconsin - Milwaukee, P.O. Box 784, Milwaukee, WI 53201, USA e-mail: munson@uwm.edu

M. C. Pimentel

Instituto de Ciências Matemáticas e de Computação, Universidade de São Paulo, Av. Trabalhador São-carlense, 400 - Centro, Caixa Postal: 668 - CEP: 13560-970, São Carlos, SP, Brasil e-mail: mgp@icmc.usp.br
} 
single representative example. The system was evaluated both with objective metrics and user testing.

A second paper addressing the digital photo domain is "Image Matting Through a Web Browser" by Lin, Wang, and Hsieh. Their NIM 2.0 system is used to specify the separation between foreground and background in a photo, usually for the purposes of placing the foreground material in some other context. NIM is the first browser-based matting application and they show that it performs high-quality matting rapidly. This work differs from research on foreground/background separation [1] because image matting is designed to extract individual foreground elements.

Scalable video coding techniques work by reducing some aspect of video quality in order to reduce the bandwidth requirements of video streams. Daronco, Roesler, Valdeni de Lima, and Balbinot present the results of experimental user studies of scalable coding in "Quality Analysis of Scalable Video Coding on Unstable Transmissions". They compared scaling based on spatial, temporal, and quality changes and also looked at whether varying the level of scaling affected user assessments. Using widely accepted metrics [3], they show that temporal scaling is considered less satisfactory and that any instability in the level of scaling also reduced user assessments.

In an invited paper titled "Communicating and Migratable Interactive Multimedia Documents", Concolato, Dufourd, Le Feuvre, Park, and Song examine how to make interactive multimedia applications suitable for dynamic migration between devices. This research is important because of the rapid adoption of sophisticated mobile devices with multimedia capabilities. As people change location, they may well change devices, too, and their applications and application state must follow them as they move. Other proposed solutions are based on widget-to-widget communication [6] or a shared or central JavaScript context [4], but this one enables cross-device exchanges more effectively.

In other research addressing mobile devices, Deigmoeller, Takebumi, Just, and Stoll consider "Contextual Cropping and Scaling of TV Productions". They are interested in adapting video that was produced for large screens to the small format screens found on mobile devices. They focus on sports productions and their system tries to automatically scale or crop the video images in order to focus on the central action in the sports event. Their system makes better use of metadata than some previous systems [8] and is less content-specific than others [2].

In the paper "XTemplate 3.0: Spatio-Temporal Semantics and Structure Reuse for Hypermedia Compositions", Ferreira dos Santos and Muchaluat-Saade describe a template language for hypermedia documents. XTemplate is intended to ease the creation of complex interactive applications such as might be created for interactive television systems. XTemplate has been applied to the declarative Nested Context Language 3.0 [7] in order to provide stronger semantics and facilitate specification reuse.

In the paper "Discrimination of media moments and media intervals: sticker-based watch-and-comment annotation", Texeira, Mello, Freitas, Santos and Pimentel describe an approach for annotating interactive broadcast video with "media stickers". They define a set of operators that can be used to apply media stickers, which are predefined media elements. Users apply the stickers in order to identify moments of interest in video programs for later review by themselves or others. A small user test is presented as an initial evaluation. This is another example of work building on the Nested Context Language [7]. 


\section{Final thoughts}

Two points should be clear from the work presented in this special issue. First, even in a well-studied area like digital still images, there is considerable room of innovative research. Many natural editing operations are still not well supported and image collections get harder to manage as they expand with the ease of taking photographs. Second, new devices and technologies always create opportunities for researchers. Mobile devices and upcoming interactive TV systems are the examples seen here.

\section{References}

1. Amri S, Barhoumi W, Zagrouba E (2009) A robust framework for joint background/foreground segmentation of complex video scenes filmed with freely moving camera. Multimed Tools Appl 46:175-205

2. Dearden A, Demiris Y, Grau O (2006) Tracking football player movement from a single moving camera using particle filters. Proceedings of the 3rd European Conference on Visual Media Production (CVMP), London. pp.29-37

3. Dumic E, Grgic S, Grgic M (2010) Comparison of HDTV formats using objective video quality measures. Multimed Tools Appl, Springer. doi:10.1007/s11042-009-0441-2

4. Jansen J, Bulterman DC (2009) SMIL State: an architecture and implementation for adaptive time-based web applications. Multimed Tools Appl 43(3):203-224. doi:10.1007/s11042-009-0270-3

5. Schaefer G (2010) A next generation browsing environment for large image repositories. Multimed Tools Appl 47(1):105-120

6. Sire S, Paquier M, Vagner A, Bogaerts J. (2009) A messaging API for interwidgets communication. In: Proceedings of the 18th international Conference on World Wide Web, Madrid, Spain, April 20-24, 2009. WWW'09. ACM, New York, NY, 1115-1116. doi:10.1145/1526709.1526884

7. Soares LFG, Rodrigues RF, Cerqueira R, Barbosa SDJ (2010) Variable and state handling in NCL. Multimed Tools Appl 50:465-489

8. Zaller J (2007) Snell \& Wilcox's Helios. http://broadcastengineering.com/RF/broadcasting_snell_wilcoxs_helios/ index.html. Accessed 26 June 2010

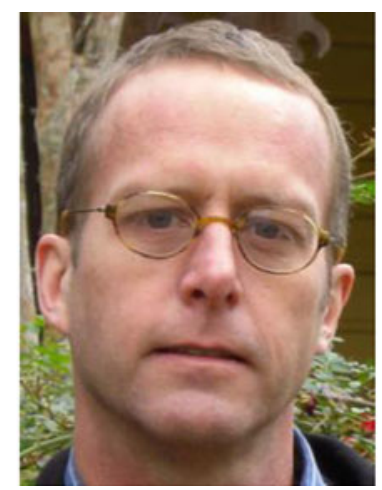

Ethan V. Munson is a Professor of Computer Science in the Department of Electrical Engineering and Computer Science at the University of Wisconsin-Milwaukee, where he is also the Director of the Multimedia Software Laboratory. He received the M.S. (1989) and Ph.D. (1994) in Computer Science from the University of California, Berkeley. His research focuses on configuration management for software and documents and on human-computer interaction. Dr. Munson is a recipient of a National Science Foundation CAREER award, as well as four NSF educational grants, and a variety of industrial funding. He is a Senior Member of ACM and member of the Brazilian Computer Society (SBC). He was Chair of ACM SIGWEB from 2006 to 2011. 


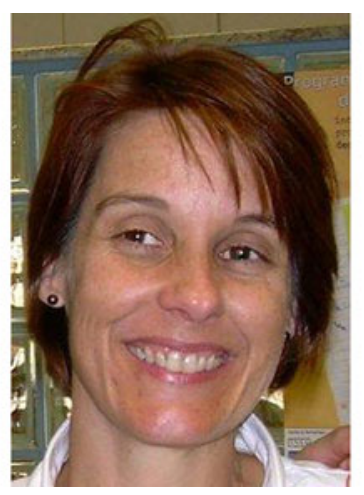

Maria da Graça C. Pimentel is a Professor of Computer Science Department at the Mathematics and Computer Science Institute of the Universidade de São Paulo at São Carlos, Brazil. She is also the head of Intermedia Lab at the same institute. Dr. Pimentel received a PhD in computer science from the University of Kent at Canterbury (UK) in 1994, and a M.Sc. in computer science from the Universidade de São Paulo (1989). Her research interests focus on ubiquitous computing as a platform for modeling, designing and implementing interactive multimedia documents. In particular, current research is related to the investigation of distributed multimodal multimedia systems and their relation to capture-based systems in general, and exploited in the context of Interactive TV in particular. She is member of ACM, IEEE and the Brazilian Computer Society (SBC). She is the treasurer of ACM SIGWEB and served as that organization's Vice-Chair from 2006 to 2011. 